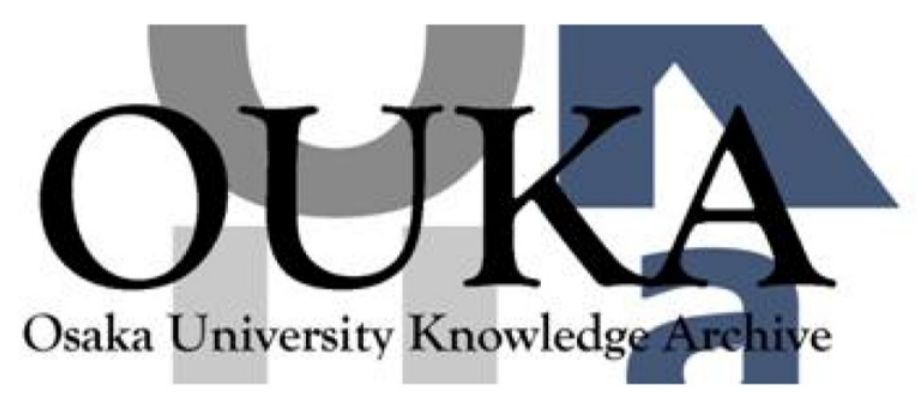

\begin{tabular}{|c|c|}
\hline Title & $\begin{array}{l}\text { Closed-form solution to a real option problem } \\
\text { with regime switching }\end{array}$ \\
\hline Author (s) & Nishihara, Michi \\
\hline Citation & Operations Research Letters. 48(6) p.703-p.707 \\
\hline Issue Date & $2020-11$ \\
\hline oaire:version & AM \\
\hline URL & https://hdl. handle. net/11094/78922 \\
\hline rights & $\begin{array}{l}\text { (- } 2020 \text { Elsevier B.V. This manuscript version is } \\
\text { made avai lable under the Creative Commons } \\
\text { Attribution-NonCommercial-NoDerivatives } 4.0 \\
\text { International license. }\end{array}$ \\
\hline Note & \\
\hline
\end{tabular}

Osaka University Knowledge Archive : OUKA

https://ir. Library. osaka-u. ac. jp/

0saka University 


\title{
Closed-form solution to a real option problem with regime switching
}

\author{
Michi NISHIHARA*, Osaka University
}

\begin{abstract}
I investigate the optimal investment timing model in which investment is feasible in only one of the two regimes, which shift at Poisson jump times. I derive the option value and investment threshold in closed forms. I also prove that some solutions in previous models are obtained as the limits of the solution. The closed-form solution can be useful as a new framework to study real option problems with the illiquidity of option exercise opportunities.
\end{abstract}

Keywords: finance; real option; investment timing; regime switching; illiquidity.

Short title: Solution to real option problem with regime switching

${ }^{*}$ Corresponding Author: Michi Nishihara.

1-7 Machikaneyama, Toyonaka, Osaka 560-0043, Japan. nishihara@econ.osaka-u.ac.jp. 


\section{Introduction}

In project valuation involving high uncertainty and managerial flexibility, the real option value (ROV) method adds value to the net present value (NPV) method (see [1]). An increasing number of papers have investigated various problems in the ROV framework (for a recent review, refer to [2]). Most of these studies presume that an option holder can exercise his/her real option at an arbitrary time, analogous to American call and put options. However, this assumption does not always hold true for real-world problems.

For example, consider a firm that will expand its business by acquiring (a certain section of) another firm. If a hostile acquisition is too costly, the firm begins by searching for a target that may potentially agree to be acquired. Only if the firm finds such a target and the negotiation and/or bidding process succeeds, the firm can acquire the target assets. Otherwise, the firm begins by searching for another target. In this case, the firm can exercise the acquisition option, not at an arbitrary time but only while it has a potential target satisfying certain conditions. Similarly, in an asset liquidation problem, a firm can sell illiquid assets at fair prices not at an arbitrary time but only while it finds a potential buyer satisfying certain conditions.

This paper captures the illiquidity of option exercise opportunities by a regime switching model. More precisely, I examine the optimal investment timing problem where investment is feasible in only one of the two regimes, which shift at Poisson jump times. The feasible regime is called the good regime, while the infeasible regime is called the bad regime. Most notably, I derive the value function and investment threshold in closed forms. Because of its simplicity (i.e., the closed-form solution), the proposed model can be useful as a new framework to study real option problems with the illiquidity of option exercise opportunities (including but not limited to acquisition and asset liquidation).

I also show the following results. The option value and investment threshold increase as the arrival rate of the good (bad) regime increases (decreases). In other words, the value of waiting increases for a project with higher liquidity of option exercise opportunities. The solution converges to that of the standard ROV model in [1] as the arrival rate of the bad (good) regime goes to zero (infinity). The solution converges to that of the optimal stopping problem constrained within Poisson jump times in [3], as the arrival rate of the bad regime goes to infinity. The solution converges to that of the ROV model in which the option is lost at the first Poisson jump time, as the arrival rate of the good regime goes to zero. The solution converges to that of the NPV model, as the arrival rates of the good and bad regimes go to zero and infinity, respectively. Thus, the closed-form solution in this paper fills the gaps in these previous models.

Lastly, I note differences from existent ROV models with regime switching. The model is regarded as a simplified version of a more general ROV model with regime switching (e.g., [4], [5], and [6]), where investment is also feasible in the bad regime. One cannot obtain a closed-form solution in the general model because the general problem is reduced to two nonlinear equations for two investment thresholds. By restricting investment in only one regime, I resolve this issue 
and reduce the problem to one linear equation for one investment threshold, which leads to the closed-form solution.

The closed-form solution has an advantage over nonclosed-form solutions in previous literature because it allows us to examine comparative statics, as wells as economic implications, without specifying parameter values. In fact, by virtue of the closed-form solution, this paper, unlike previous papers, clarifies the limiting results and the relation among the previous models. In particular, I show that the closed-form solution in the regime switching model nests that of [3], although previous papers are unaware of this link. In terms of application, this paper is more relevant to the illiquidity of searching and matching than macroeconomic shocks, although previous papers focus shocks from macroeconomic conditions by regime switching.

\section{Model Setup}

I examine an ROV model with regime switching as follows. Consider a firm that has an option to invest in a project. In one of the regimes (called the good regime), the firm can invest in the project at an arbitrary time, whereas in the other regime (called the bad regime), the firm is not allowed to invest. The bad regime shifts to the good regime at a Poisson jump time with the arrival rate $\lambda(>0)$, whereas the good regime shifts to the bad regime at a Poisson jump time with the arrival rate $\eta(>0)$. The investment project requires the initial investment cost $I$ and generates a profit $X(t)$, which follows a geometric Brownian motion

$$
\mathrm{d} X(t)=\mu X(t) \mathrm{d} t+\sigma X(t) \mathrm{d} B(t)(t>0), \quad X(0)=x,
$$

where $B(t)$ denotes the standard Brownian motion defined in a filtered probability space $\left(\Omega, \mathcal{F}, \mathbb{P},\left\{\mathcal{F}_{t}\right\}\right)$ and $\mu, \sigma(>0)$ and $x(>0)$ are constants. For the model tractability, I assume that $B(t)$ is independent of the Poisson processes. A positive constant $r$ denotes the discount rate, and for convergence I assume that $r>\mu$.

I denote by $V(x)$ and $U(x)$ the option values in the bad and good regimes, respectively. The regime-switching model can be regarded as a simplified version of more general models by [4] [5], and [6], where investment is feasible in any regime.

\section{Model Solution}

Following [4], I can solve the model. I presume that the firm's optimal policy is a threshold policy expressed as $\inf \left\{t \geq 0 \mid X(t) \geq x^{*}\right.$ in the good regime $\}$. Note that the optimality of the threshold policy under more general conditions is shown in [6]. Then, the value functions $V(x)$ and $U(x)$ satisfy ordinary differential equations (ODEs)

$$
\begin{array}{cc}
\mu x V^{\prime}(x)+0.5 \sigma^{2} x^{2} V^{\prime \prime}(x)+\lambda(U(x)-V(x))=r V(x), & \left(x<x^{*}\right), \\
\mu x V^{\prime}(x)+0.5 \sigma^{2} x^{2} V^{\prime \prime}(x)+\lambda(x-I-V(x))=r V(x), & \left(x>x^{*}\right), \\
\mu x U^{\prime}(x)+0.5 \sigma^{2} x^{2} U^{\prime \prime}(x)+\eta(V(x)-U(x))=r U(x) & \left(x<x^{*}\right),
\end{array}
$$


where $\lambda(U(x)-V(x))$ in (1) and $\lambda(x-I-V(x))$ in (2) reflect that $V(x)$ changes to $U(x)$ and $x-I$ when the bad regime shifts to the good regime, whereas $\eta(V(x)-U(x))$ in (3) means that $U(x)$ changes to $V(x)$ when the good regime switches to the bad regime.

The boundary conditions are $\lim _{x \rightarrow 0} V(x)=0, \lim _{x \rightarrow \infty} V(x) / x<\infty, \lim _{x \rightarrow 0} U(x)=0, U(x)=$ $x-I\left(x \geq x^{*}\right)$, and the continuous differentiability of $V(x)$ and $U(x)$ at $x=x^{*}$. The first four boundary conditions are trivial. The continuous differentiability of $U(x)$ is the smooth pasting condition in [1], whereas the continuous differentiability of $V(x)$ follows from Theorem 4.4.9 in [7] (see [4]). By solving ODE (1)-(3), I have the following proposition, where I define, for $y>0$,

$$
\begin{aligned}
& \beta_{y}=0.5-\frac{\mu}{\sigma^{2}}+\sqrt{\left(\frac{\mu}{\sigma^{2}}-0.5\right)^{2}+\frac{2(r+y)}{\sigma^{2}}}(>1), \\
& \gamma_{y}=0.5-\frac{\mu}{\sigma^{2}}-\sqrt{\left(\frac{\mu}{\sigma^{2}}-0.5\right)^{2}+\frac{2(r+y)}{\sigma^{2}}}(<0),
\end{aligned}
$$

and for simplicity, I denote $\beta=\beta_{0}$ and $\gamma=\gamma_{0}$.

\section{Proposition 1}

$$
\begin{aligned}
& V(x)= \begin{cases}A_{1} x^{\beta}-\frac{\lambda}{\eta} A_{2} x^{\beta_{\lambda+\eta}} & \left(x<x^{*}\right), \\
\frac{\lambda x}{r+\lambda-\mu}-\frac{\lambda I}{r+\lambda}+A_{3} x^{\gamma_{\lambda}} & \left(x \geq x^{*}\right),\end{cases} \\
& U(x)= \begin{cases}A_{1} x^{\beta}+A_{2} x^{\beta_{\lambda+\eta}} & \left(x<x^{*}\right), \\
x-I & \left(x \geq x^{*}\right),\end{cases}
\end{aligned}
$$

where the investment threshold $x^{*}$ and coefficients $A_{1}, A_{2}, A_{3}$ are

$$
\begin{aligned}
x^{*} & =\frac{\left(\beta(r+\lambda)\left(\beta_{\lambda+\eta} \eta+\lambda\left(\beta_{\lambda+\eta}-\gamma_{\lambda}\right)\right)-\gamma_{\lambda} \eta\left(\beta_{\lambda+\eta} r+\beta \lambda\right)\right)(r+\lambda-\mu) I}{\left(\lambda(\beta-1)\left(\beta_{\lambda+\eta}-\gamma_{\lambda}\right)(r+\lambda+\eta-\mu)+\eta\left(\beta-\gamma_{\lambda}\right)\left(\beta_{\lambda+\eta}-1\right)(r-\mu)\right)(r+\lambda)}, \\
A_{1} & =\frac{\left(\beta_{\lambda+\eta}-1\right) x^{*}-\beta_{\lambda+\eta} I}{\left(\beta_{\lambda+\eta}-\beta\right) x^{* \beta}}, \\
A_{2} & =\frac{\beta I-(\beta-1) x^{*}}{\left(\beta_{\lambda+\eta}-\beta\right) x^{* \beta_{\lambda+\eta}}}, \\
A_{3} & =\frac{1}{x^{* \gamma_{\lambda}}}\left(A_{1} x^{* \beta}-\frac{\lambda}{\eta} A_{2} x^{* \beta_{\lambda+\eta}}-\frac{\lambda x^{*}}{r+\lambda-\mu}+\frac{\lambda I}{r+\lambda}\right) .
\end{aligned}
$$

Proof I define $f(x)=U(x) \lambda / \eta+V(x)$ and $g(x)=U(x)-V(x)$. By (1) and (3), I have

$$
\begin{aligned}
\mu x f^{\prime}(x)+0.5 \sigma^{2} x^{2} f^{\prime \prime}(x) & =r f(x), & \left(x<x^{*}\right), \\
\mu x g^{\prime}(x)+0.5 \sigma^{2} x^{2} g^{\prime \prime}(x)+(\lambda+\eta) g(x) & =r g(x), & \left(x<x^{*}\right) .
\end{aligned}
$$

A general solution to ODE (12) with the boundary condition $\lim _{x \rightarrow 0} f(x)=0$, which follows from $\lim _{x \rightarrow 0} V(x)=\lim _{x \rightarrow 0} U(x)=0$, is expressed as $B_{1} x^{\beta}$, where $B_{1}$ is an indefinite coefficient. A general solution to ODE (13) with the boundary condition $\lim _{x \rightarrow 0} g(x)=0$, which follows from $\lim _{x \rightarrow 0} V(x)=\lim _{x \rightarrow 0} U(x)=0$, is expressed as $B_{2} x^{\beta_{\lambda+\eta}}$, where $B_{2}$ is an indefinite coefficient. Then, I have the expressions (6) and (7) for $x<x^{*}$ by taking $A_{i}=B_{i} \eta /(\lambda+\eta)(i=1,2)$. A general solution to ODE (2) with the boundary condition $\lim _{x \rightarrow \infty} V(x) / x<\infty$, is expressed as (6) for $x \geq x^{*}$, where $A_{3}$ is an indefinite coefficient. 
Below, I will derive $x^{*}$ and $A_{i}(i=1,2,3)$ by the continuous differentiability of $V(x)$ and $U(x)$ at $x=x^{*}$. By the continuous differentiability of $U(x)$ at $x=x^{*}$, I have

$$
\begin{aligned}
A_{1} x^{* \beta}+A_{2} x^{* \beta_{\lambda+\eta}} & =x^{*}-I, \\
\beta A_{1} x^{* \beta}+\beta_{\lambda+\eta} A_{2} x^{* \beta_{\lambda+\eta}} & =x^{*},
\end{aligned}
$$

which lead to

$$
\begin{aligned}
A_{1} x^{* \beta} & =\frac{\left(\beta_{\lambda+\eta}-1\right) x^{*}-\beta_{\lambda+\eta} I}{\beta_{\lambda+\eta}-\beta}, \\
A_{2} x^{* \beta_{\lambda+\eta}} & =\frac{(1-\beta) x^{*}+\beta I}{\beta_{\lambda+\eta}-\beta} .
\end{aligned}
$$

By the continuous differentiability of $V(x)$ at $x=x^{*}$, I have

$$
\begin{aligned}
A_{1} x^{* \beta}-\frac{\lambda}{\eta} A_{2} x^{* \beta_{\lambda+\eta}} & =\frac{\lambda x^{*}}{r+\lambda-\mu}-\frac{\lambda I}{r+\lambda}+A_{3} x^{* \gamma_{\lambda}}, \\
\beta A_{1} x^{* \beta}-\frac{\lambda \beta_{\lambda+\eta}}{\eta} A_{2} x^{* \beta_{\lambda+\eta}} & =\frac{\lambda x^{*}}{r+\lambda-\mu}+\gamma_{\lambda} A_{3} x^{* \gamma_{\lambda}},
\end{aligned}
$$

which lead to

$$
\left(\beta-\gamma_{\lambda}\right) A_{1} x^{* \beta}-\frac{\lambda\left(\beta_{\lambda+\eta}-\gamma_{\lambda}\right)}{\eta} A_{2} x^{* \beta_{\lambda+\eta}}=\frac{\lambda\left(1-\gamma_{\lambda}\right) x^{*}}{r+\lambda-\mu}+\frac{\lambda \gamma_{\lambda} I}{r+\lambda} .
$$

By substituting (14) and (15) into (18), I have

$$
\begin{aligned}
\frac{\left(\beta-\gamma_{\lambda}\right)\left(\left(\beta_{\lambda+\eta}-1\right) x^{*}-\beta_{\lambda+\eta} I\right)}{\beta_{\lambda+\eta}-\beta}-\frac{\lambda\left(\beta_{\lambda+\eta}-\gamma_{\lambda}\right)\left((1-\beta) x^{*}+\beta I\right)}{\eta\left(\beta_{\lambda+\eta}-\beta\right)} & =\frac{\lambda\left(1-\gamma_{\lambda}\right) x^{*}}{r+\lambda-\mu}+\frac{\lambda \gamma_{\lambda} I}{r+\lambda} \\
\left(\frac{\eta\left(\beta-\gamma_{\lambda}\right)\left(\beta_{\lambda+\eta}-1\right)+\lambda(\beta-1)\left(\beta_{\lambda+\eta}-\gamma_{\lambda}\right)}{\eta\left(\beta_{\lambda+\eta}-\beta\right)}-\frac{\lambda\left(1-\gamma_{\lambda}\right)}{r+\lambda-\mu}\right) x^{*} & =\left(\frac{\eta \beta_{\lambda+\eta}\left(\beta-\gamma_{\lambda}\right)+\lambda \beta\left(\beta_{\lambda+\eta}-\gamma_{\lambda}\right)}{\eta\left(\beta_{\lambda+\eta}-\beta\right)}\right. \\
& \left.+\frac{\lambda \gamma_{\lambda}}{r+\lambda}\right) I
\end{aligned}
$$

by which I can derive $x^{*}$ in (8). By (14), (15), and (16), I can derive $A_{1}, A_{2}$, and $A_{3}$ in (9), (10), and (11), respectively. The proof is complete.

In the good regime, the firm invests in the project at time $t$ satisfying $X(t) \geq x^{*}$. The upper equation in (7) stands for the value of waiting when the option value is higher than the investment payoff, i.e, $U(x)>x-I$. The lower equation in (7) equals the investment payoff. The upper equation in (6) stands for the value of waiting when the option value is higher than the investment payoff. The lower equation in (6) stands for the value of waiting, when the option value is lower than the investment payoff. In the bad regime, for $X(t) \geq x^{*}$, the firm wishes to invest but has to wait for the good regime arrival. The first and second terms of the lower equation in (6) stand for the expected payoff of investing whenever the good regime arrives. The last term $A_{3} x^{\gamma_{\lambda}}$ stands for the value of the option to change the policy when $X(t)$ falls below $x^{*}$.

Notably, in Proposition 1, the value function and investment threshold are derived in closed forms. I can easily extend the closed-form solution into those of the optimal investment and financing models by [8], [9], and [10], although I omit the details. Models with closed-form solutions (e.g., models of [1]) are dominant in the ROV literature primarily because closed-form solutions enable us to study comparative statics and economic implications regardless of parameter values. In 
this sense, the closed-form solution of Proposition 1 has an advantage over nonclosed-form solutions of [4] and [6], although they study more general models allowing investment in any regime.

Below, I explain how the exercise assumption addresses the difficulties in the previous papers. In a general model allowing investment in the bad regime (say, the payoff $b x-I_{b}(<x-I)$ ), ODEs (1)-(3) are unchanged, but the boundary condition to ODE (2) becomes the continuous differentiability condition at the investment threshold in the bad regime (say, $x_{b}^{*}\left(>x^{*}\right)$ ) rather than $\lim _{x \rightarrow \infty} V(x) / x<\infty$. Then, $V(x)$ has an extra term $A_{4} x^{\beta_{\lambda}}$ for $x \in\left[x^{*}, x_{b}^{*}\right)$ and becomes $b x-I_{b}$ for $x \geq x_{b}^{*}$. The equations are reduced to two nonlinear equations for $x^{*}$ and $x_{b}^{*}$ rather than a linear equation for $x^{*}$ like (19) because (16) and (17) have extra terms $A_{4} x^{\beta_{\lambda}}$ and $\beta_{\lambda} A_{4} x^{\beta_{\lambda}}$, respectively. The two nonlinear equations cannot be solved analytically. In this paper, by excluding investment in the bad regime, I reduce the equations to the linear equation (19), which leads to the closed-form solution. This derivation technique has never been seen in previous literature.

By virtue of the closed-form solution in Proposition 1, I can show the following proposition.

Proposition $2 V(x), U(x)$, and $x^{*}$ monotonically increase (decrease) in $\lambda(\eta)$. As $\eta \rightarrow 0, U(x)$ and $x^{*}$ converge to those (denoted by $V^{i}(x)$ and $x^{i}$ ) of the standard ROV model in [1]. As $\eta \rightarrow \infty$, $V(x), U(x)$, and $x^{*}$ converge to those (denoted by $V^{j}(x), U^{j}(x)$, and $x^{j}$ ) of [3] who study the optimal stopping problem constrained within Poisson jump times. As $\lambda \rightarrow 0, U(x)$ and $x^{*}$ converge to those (denoted by $V^{k}(x)$ and $x^{k}$ ) of the ROV model in which the investment option is killed at the first Poisson jump time with the arrival rate $\eta$. As $\lambda \rightarrow \infty, U(x)$ and $x^{*}$ converge to $V^{i}(x)$ and $x^{i}$. As $\lambda \rightarrow 0$ and $\eta \rightarrow \infty, U(x)$ and $x^{*}$ converge to those (denoted by $V^{n}(x)$ and $x^{n}$ ) of the NPV model.

Proof By definition of the problem, $V(x)$ and $U(x)$ monotonically increase (decrease) in $\lambda(\eta)$. The monotonicity of $x^{*}$ follows from the monotonicity of $U(x)$ and $x^{*}=\min \{x \geq 0 \mid U(x)=x-I\}$.

By (8), I have

$$
\begin{aligned}
\lim _{\eta \rightarrow 0} x^{*} & =\frac{\left(\beta(r+\lambda) \lambda\left(\beta_{\lambda}-\gamma_{\lambda}\right)(r+\lambda-\mu) I\right.}{\lambda(\beta-1)\left(\beta_{\lambda}-\gamma_{\lambda}\right)(r+\lambda-\mu)(r+\lambda)} \\
& =\frac{\beta I}{\beta-1}\left(=x^{i}\right),
\end{aligned}
$$

where (20) equals the investment threshold $x^{i}$ of the standard ROV model in [1]. By substituting (20) into (10), I have $\lim _{\eta \rightarrow 0} A_{2}=0$. Then, by (9), for $x<x^{i}$, I have

$$
\begin{aligned}
\lim _{\eta \rightarrow 0} U(x) & =\left(\frac{x}{x^{i}}\right)^{\beta} \frac{1}{\beta_{\lambda}-\beta}\left(\frac{\left(\beta_{\lambda}-1\right) \beta I}{\beta-1}-\beta_{\lambda} I\right) \\
& =\left(\frac{x}{x^{i}}\right)^{\beta} \frac{I}{\beta-1}\left(=V^{i}(x)\right),
\end{aligned}
$$

where (21) equals the option value of the standard ROV model.

Next, suppose $\eta \rightarrow \infty$. Note that $\lim _{\eta \rightarrow \infty} \beta_{\lambda+\eta}=\infty$ follows from (4). Then, by (8)-(11), I 
have

$$
\begin{aligned}
\lim _{\eta \rightarrow \infty} x^{*} & =\frac{\left(\beta(r+\lambda)-\gamma_{\lambda} r\right)(r+\lambda-\mu) I}{\left(\lambda(\beta-1)+\left(\beta-\gamma_{\lambda}\right)(r-\mu)\right)(r+\lambda)}\left(=x^{j}\right), \\
\lim _{\eta \rightarrow \infty} A_{1} & =\frac{x^{j}-I}{x^{j \beta}}, \\
\lim _{\eta \rightarrow \infty} A_{2} & =0, \\
\lim _{\eta \rightarrow \infty} A_{3} & =\frac{1}{x^{j \gamma_{\lambda}}}\left(\frac{(r-\mu) x^{j}}{r+\lambda-\mu}-\frac{r I}{r+\lambda}\right),
\end{aligned}
$$

where (22) equals the threshold $x^{j}$ for the optimal stopping problem constrained within Poisson jump times (i.e., (15) in [3]). By (22)-(25), I can see that $\lim _{\eta \rightarrow 0} V(x)$ and $\lim _{\eta \rightarrow 0} U(x)$ also agree with the value functions $V^{j}(x)$ and $U^{j}(x)$ of the problem (i.e., (13), (14), and (16) in [3]).

Now, suppose $\lambda \rightarrow 0$. By (8), I have

$$
\begin{aligned}
\lim _{\lambda \rightarrow 0} x^{*} & =\frac{\left(\beta r \beta_{\eta} \eta-\gamma \eta \beta_{\eta} r\right)(r-\mu) I}{\eta(\beta-\gamma)\left(\beta_{\eta}-1\right)(r-\mu) r}, \\
& =\frac{\beta_{\eta} I}{\beta_{\eta}-1}\left(=x^{k}\right),
\end{aligned}
$$

where (26) equals the threshold $x^{k}$ of the ROV model in which the option is lost at the first Poisson jump time with the arrival rate $\eta$. By substituting (26) into (9), I have $\lim _{\lambda \rightarrow 0} A_{1}=0$. Then, for $x<x^{k}$, I have

$$
\begin{aligned}
\lim _{\lambda \rightarrow 0} U(x) & =\left(\frac{x}{x^{k}}\right)^{\beta_{\eta}} \frac{1}{\beta_{\eta}-\beta}\left(\beta I-\frac{(\beta-1) \beta_{\eta} I}{\beta_{\eta}-1}\right) \\
& =\left(\frac{x}{x^{k}}\right)^{\beta_{\eta}} \frac{I}{\beta_{\eta}-1}\left(=V^{k}(x)\right),
\end{aligned}
$$

where (27) equals the corresponding option value.

Note that $\lim _{\lambda \rightarrow \infty} \beta_{\lambda+\eta} / \lambda=\lim _{\lambda \rightarrow \infty} \gamma_{\lambda} / \lambda=0$ follows from (4) and (5), respectively. By taking $\lambda \rightarrow \infty$ in (8)-(11), I can show

$$
\begin{aligned}
\lim _{\lambda \rightarrow \infty} x^{*} & =\frac{\beta I}{\beta-1}\left(=x^{i}\right), \\
\lim _{\lambda \rightarrow \infty} A_{1} & =\frac{x^{i}-I}{x^{i \beta}}, \\
\lim _{\lambda \rightarrow \infty} A_{2} & =0 .
\end{aligned}
$$

By taking $\eta \rightarrow \infty$ in (26) and (27), I have

$$
\begin{aligned}
\lim _{\substack{\lambda \rightarrow 0 \\
\eta \rightarrow \infty}} x^{*} & =(r-\mu) I\left(=x^{n}\right), \\
\lim _{\substack{\lambda \rightarrow 0 \\
\eta \rightarrow \infty}} U(x) & =\left\{\begin{array}{ll}
0 & (x<I), \\
x-I & (x \geq I),
\end{array} \quad\left(=V^{n}(x)\right)\right.
\end{aligned}
$$

which agree with the investment threshold and value function of the NPV model. The proof is complete.

Proposition 2 shows that the model solution nests several solutions in the previous models. With a higher $\lambda$ (or a lower $\eta$ ), the firm stays in the good regime longer, and hence, the value of 
deferring investment increases. Then, the option value and investment threshold increase with a higher $\lambda$ (or a lower $\eta$ ). For $\lambda \rightarrow \infty$ or $\eta \rightarrow 0$, the solution converges to the standard ROV solution in $[1]$.

With a higher $\eta$, the good regime ends sooner, and hence, the value of deferring investment decreases. As the good regime immediately changes to the bad regime for $\eta \rightarrow \infty$, the firm has to decide whether or not to invest as soon as the good regime arrives. Then, the solution converges to that of the optimal stopping problem constrained within Poisson jump times in [3].

With a lower $\lambda$, the bad regime is less likely to change to the good regime. For $\lambda \rightarrow 0$, the investment option will never return once the firm faces the bad regime. Then, the solution converges to that of the ROV model with the possibility that the option disappears. Especially for $\lambda \rightarrow 0$ and $\eta \rightarrow \infty$, the good regime immediately changes to the bad regime and will never return. Hence, the solution converges to that of the NPV model in which the firm has to decide whether to invest now or never.

The limiting results are not shown in the regime switching literature (e.g., [4] and [6]) because nonclosed-form solutions prevent us from calculating the limits analytically. In particular, no study on the ROV models with regime switching is aware of the relation with [3]. The two strands of research have developed independently. This paper first shows that the solution of [3] is a limiting solution in the ROV model with regime switching. Indeed, the closed-form solution in Proposition 1 generalizes that of [3].

Lastly, I see the results in numerical examples. The baseline parameters are set at $r=0.07, \mu=$ $0.05, \sigma=0.2, I=1, x=1, \lambda=1$, and $\eta=1$. Then, I have $V(x)=0.5205, U(x)=0.5205, x^{*}=$ 4.522 in the current model, $V^{i}(x)=0.522, x^{i}=4.766$ in the standard ROV model, $V^{j}(x)=U^{j}(x)=$

$0.5194, x^{j}=4.044$ in the model constrained within Poisson jump times, $V^{k}(x)=0.0603, x^{k}=1.179$ in the ROV model killed by the first Poisson jump time, and $V^{n}(x)=0, x^{n}=1$ in the NPV model. Note that $U(x)-V(x)$ is approximately $2 \times 10^{-12}$ for $x=1$. Figure 1 shows $U(x)$ and $x^{*}$ for varying levels of $\lambda$ and $\eta$. I omit depicting $V(x)$ because differences between $V(x)$ and $U(x)$ are quite small and invisible in most areas. I can see that the option value and investment threshold increase with a higher $\lambda$ (or a lower $\eta$ ). I can also check the limiting results in Proposition 2.

\section{Acknowledgments}

The author thanks the editor, an anonymous referee, Junichi Imai, Takashi Shibata, and Kazutoshi Yamazaki for helpful comments. The paper was presented the SMTDA 2018 conference in Crete, ISERD 2018 conference in Taipei, and ORSJ 2018 spring conference in Tokyo. The author thanks the participants for helpful feedbacks. This work was supported by the JSPS KAKENHI (Grant number 20K01769, JP17K01254, JP17H02547). 

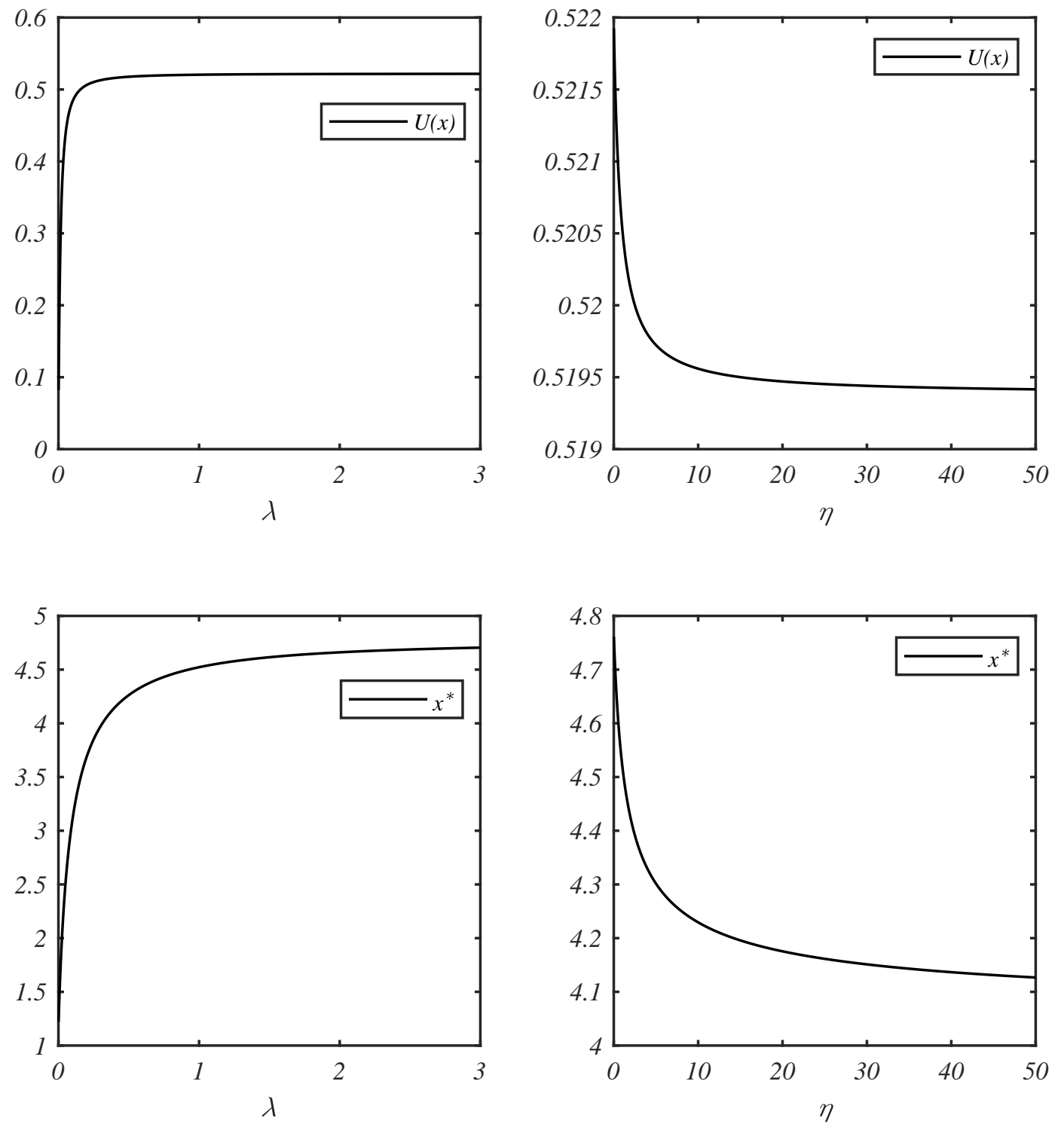

Figure 1: $U(x)$ and $x^{*}$ with respect to $\lambda$ and $\eta$. 


\section{References}

[1] A. Dixit, R. Pindyck, Investment Under Uncertainty, Princeton University Press, Princeton, 1994.

[2] L. Trigeorgis, A. Tsekrekos, Real options in operations research: A review, European Journal of Operational Research 270 (2018) 1-24.

[3] P. Dupuis, H. Wang, Optimal stopping with random intervention times, Advances in Applied Probability 34 (2002) 141-157.

[4] D. Hackbarth, J. Miao, E. Morellec, Capital structure, credit risk, and macroeconomic conditions, Journal of Financial Economics 82 (2006) 519-550.

[5] R. Elliott, H. Miao, J. Yu, Investment timing under regime switching, International Journal of Theoretical and Applied Finance 12 (2009) 443-463.

[6] A. Bensoussan, Z. Yan, G. Yin, Threshold-type policies for real options using regime-switching models, SIAM Journal on Financial Mathematics 3 (2012) 667-689.

[7] I. Karatzas, S. Shreve, Brownian Motion and Stochastic Calculus, Springer, New York, 1998.

[8] S. Sundaresan, N. Wang, Investment under uncertainty with strategic debt service, American Economic Review, Papers \& Proceedings 97 (2007) 256-261.

[9] T. Shibata, M. Nishihara, Investment timing under debt issuance constraint, Journal of Banking and Finance 36 (2012) 981-991.

[10] T. Shibata, M. Nishihara, Investment timing, debt structure, and financing constraints, European Journal of Operational Research 241 (2015) 513-526. 